\title{
Angle of Incidence and Polarization Insensitive Ultra-Thin Ultra Wide-Band Metamaterial Absorber based on Novel Non-Linearity in Unit Cell Design
}

\author{
Alkesh Agrawal (10, Mukul Misra (1) \\ Department of Electronics \& Communication Engineering, Shri Ramswaroop Memorial University, \\ Barabanki, India \\ alkesh.agrawal26@gmail.com,mukul.katyayan@gmail.com
}

\begin{abstract}
A new design of an ultra wide-band metamaterial absorber (MMA), with appreciable high absorptance insensitive to angle of incidence and polarization angle is presented in the manuscript. The MMA structure consists of three concentric rings with non-linear variation in the spacing and the thickness of the rings is controlled by a single parameter. The idea of utilizing nonlinear variation based unit cell resulted in high absorptance ultra wide-band frequency spectrum in $X$-band. The proposed MMA design is three layered structure. The metamaterial nature of the proposed device is explained by simulated values of $Z_{\text {eff }}(f), \mu_{\text {eff }}(f)$, $\varepsilon_{\text {eff }}(f)$, and $\eta_{\text {eff }}(f)$. The FWHM bandwidth of proposed MMA is 6.32 GHz (7.36 GHz-13.68 GHz). The fabricated MMA is ultra-thin with thickness of $\lambda_{0} / 15.3$ at centre frequency $9.8 \mathrm{GHz}$. The experimental results show absorptance greater than $99 \%$ at $7.9 \mathrm{GHz}$ and 11.7 $\mathrm{GHz}$, covering entire $X$-band range makes this MMA appropriate for providing stealth technology for defense equipments by reducing radar cross section $(\mathrm{RCS})$.
\end{abstract}

Index Terms - absorptance, microwave absorber, reflectance, resonator, wideband.

\section{INTRODUCTION}

Metamaterials (MMs) are the structures not found in nature therefore, these materials are engineered artificially to derive unusual properties from the dimensions of the structure, repetitive patterns in the structure, design, and spatial arrangement of the structure [1]. These materials are found capable of controlling propagation of electromagnetic (EM) waves [2]. The materials that can exhibit negative refractive index were explained by Veselago [1] in 1968. Pendry was the first to practically identify the left-handed materials exhibiting negative values of $\mu(f)$ and $\varepsilon(f)$. Combination of periodic array of split ring resonators and micro-structured thin wires was suggested and implemented to design metamaterials [3]-[5]. In recent years, the research in MMs have significantly increased due to their ability to create artificial electromagnetic responses with promising implementations such as perfect lens [6], [7] in devices with negative refractive index, cloaking [8] in devices for hiding objects, MM based compact patch antennas [9] in radiating devices, $\mathrm{MM}$ based $\mathrm{THz}$ waveguide [10] and 
Journal of Microwaves, Optoelectronics and Electromagnetic Applications, Vol. 20, No. 2, September 2021

DOI: http://dx.doi.org/10.1590/2179-10742021v20i31207

metamaterial absorbers (MMAs) [11]-[24] in absorbing devices. The recent research trends for MM based microwave absorbers exhibit nearly unity absorption [11], millimeter scale dimensions, single to multiband absorptance peaks [11]-[24], insensitive to polarization angle and wide angle of incidence [17]-[21] found applications in photo-detectors [25], wireless LAN [26], micro-electronic photonic circuits [27], MM spectroscopy and imaging [28].

In order to have wide range of applications researchers go for multi-band and wide-band MMAs. To achieve multi-band and wide-band absorptance in desired range of frequencies, multi-layer design [29], lumped elements [30], scaled design [21], [22] are proposed which makes the MMA structure complicated for fabrication and also results in increased size.

The concept of non-linear structure is often utilized to achieve the wide-band performance of antenna in microwave and $\mathrm{THz}$ frequency range [31], [32]. Keeping it in mind, we proposed a unique unit cell structure of three concentric rings with non-linear variation in the width and the spacing of the rings controlled by a single parameter in the MMA. The optimized MMA is fabricated and experimentally tested. The new concept of non-linearity in MMA resulted in high absorptance ultra wide-band.

\section{STRUCTURE, DESIGN, AND SIMULATION OF METAMATERIAL ABSORBER}

Conventional MPA [11] consisted of three layers. MMAs are complex valued with $\varepsilon(f)=\varepsilon_{1}+\mathrm{j} \varepsilon_{2}$ and $\mu(f)=\mu_{1}+\mathrm{j} \mu_{2}$ where real values contribute to propagation of EM waves in the material and imaginary values (loss components) contribute to attenuation of EM waves resulting in high absorption of EM waves [33]. The shape of unit cell and its periodicity in MMA structures are engineered to have impedance matched with free space to have minimum reflectivity and maximum absorption [17]-[22]. The proposed MMA structure consists of a symmetric three layered structure with triple concentric rings at the top, FR-4 dielectric lossy layer $\left(\varepsilon_{\mathrm{r}}=4.4, \tan \delta=0.02\right)$ in the middle, and copper laminated layer at the bottom as shown in Fig. 1(a). MMA structure unit cell consists of triplet concentric circular rings in which two rings, middle ring and outer most ring are splitted and innermost ring is continuous. The concept of non-linearity in terms of log-periodicity is implemented in the design of antennas to have wider bandwidth with high gain [29], [30]. The same concept of non-linearity is implemented in the proposed design of MMA as described by the quadratic equations controlling the radius, the width and the spacing between the rings: $r_{3}=r_{1} * x_{1} \mathrm{~mm}, r_{5}=r_{1} * x_{1}^{2} \mathrm{~mm}, r_{2}=r_{1} * x_{1}+r_{1} *$ $x_{1} / 2 \mathrm{~mm}, r_{4}=r_{1} * x_{1}^{2}+r_{1} *\left(x_{1}^{2} / 2\right) \mathrm{mm}$. The element $x_{1}$ controls the design of the proposed MMA and adds non-linearity which gives uniqueness to the proposed design of MMA. In order to obtain maximum bandwidth with high absorptance in $X$-band the values of $a, b, r_{1}$, and $x_{1}$ are optimized to 7 $\mathrm{mm}, 7 \mathrm{~mm}, 3.15 \mathrm{~mm}$, and $0.5 \mathrm{~mm}$ respectively. CST Microwave Studio suite is used to simulate unit cell of MMA for normally incident electromagnetic waves with 16,450 tetrahedral meshes. Along the direction of propagation ( $z$-direction), open bounding conditions are applied and along $x$-direction and $y$ - direction unit cell periodic bounding conditions are applied [19], [20]. The absorptance is calculated using (1) [12]-[14], where $S_{11}(f)$ is the reflectance and $S_{21}(f)$ is the transmittance, 


$$
\mathrm{A}(f)=1-\left|\mathrm{S}_{11}(f)\right|^{2}-\left|\mathrm{S}_{21}(f)\right|^{2}
$$

$S_{21}(f)=0$ due to copper laminated bottom layer of MMA structure thereby minimizing reflectivity and maximizing the absorptance.

The wide-band MMA has absorptance of greater than $99 \%$ at $7.9 \mathrm{GHz}$ and $11.7 \mathrm{GHz}$. The two absorption bands merged to achieve ultra wide-band of FWHM bandwidth of $6.32 \mathrm{GHz}(7.36 \mathrm{GHz}$ 13.68 GHz) for the normal incidence of EM waves shown in Fig. 2. The non-linear variation in the spacing between the rings and the width of the rings resulted in the high absorptance for wide range of absorption bands. The top surface of the proposed MMA is engineered in terms of $2 \mathrm{D}$ periodic array of concentric ring resonators with impedance matched conditions. These ring resonators are the active regions for absorption of the incident electromagnetic radiation (EMR). Each ring resonator contributes for a specific absorption band in MMA that depends on the optimized dimensions of the ring resonator. The portion of the MMA with outer most ring resonator contributes maximum for absorption at lower frequency band where as for higher absorption band the portion of the MMA that contributes for maximum absorption are other two inner ring resonators. With non-linearity in the proposed MMA unit cell design, there is strong coupling between the ring resonators under perfectly impedance matched conditions as a result the absorption band contributed by each ring resonator got merged with each other and resulted in wide-band absorption. The simulated reflection coefficients and absorptance is shown in Fig. 2.

Surface current and field distributions are investigated at all the absorptance peaks to better understand the absorption mechanism of normally incident EMR as shown in Fig. 3, 4, and 5. When an EM wave is incident along the axis of the Split Ring Resonator (SRR), surface currents are induced along the shape of the SRR that depends on the resonant characteristics of SRR. The induced surface currents produce $H$-field that either enhances or opposes the incident field. The splits in the SRR play an important role in making the SRR to resonate at wavelength much larger than ring diameter. The split of inner concentric ring is responsible for generating high capacitance between the concentric rings and curtailing the resonance frequency and confining the $E$-field. The dimensions of SRR being much lesser than the wavelength of incident EMR reduces the radiation losses and makes $\mathrm{Q}$ factor much high [4]. 


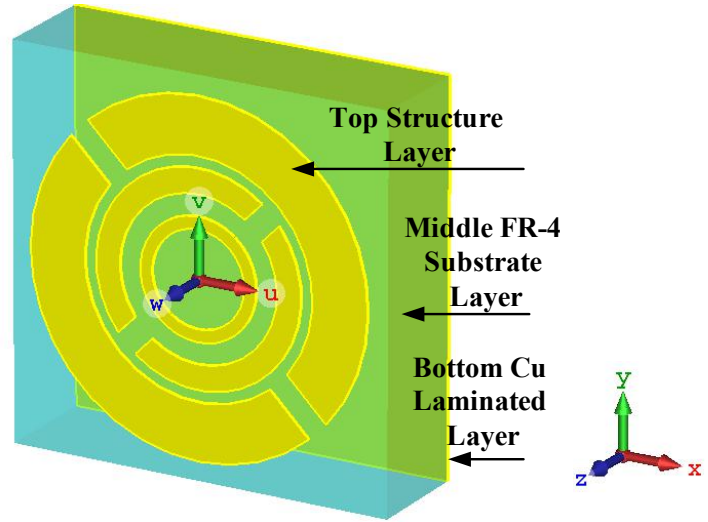

(a)

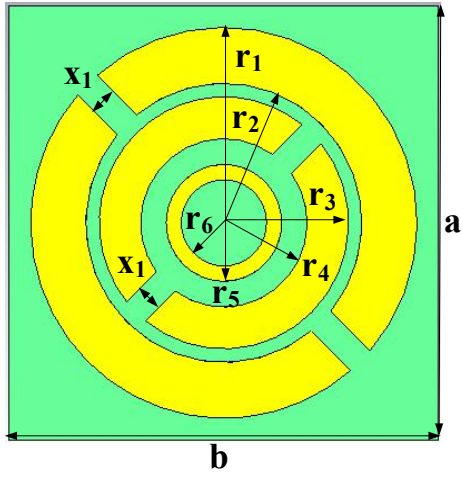

(b)

Fig. 1. (a) Three dimensional view (b) Dimensions of the rings of unit cell.

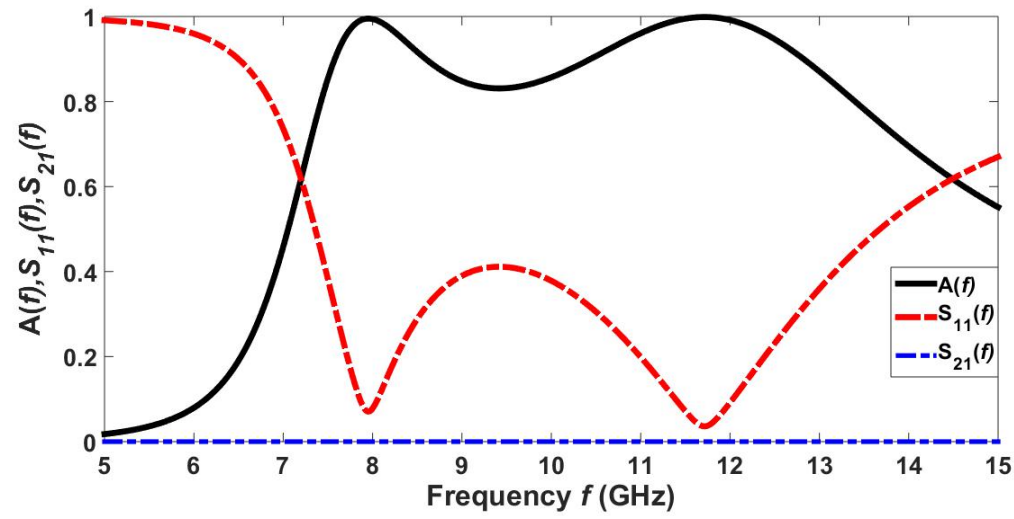

Fig. 2. Absorptance, $A(f)$, Reflection coefficient, $S_{11}(f)$, and Transmittance, $S_{21}(f)$ of the proposed MMA structure.

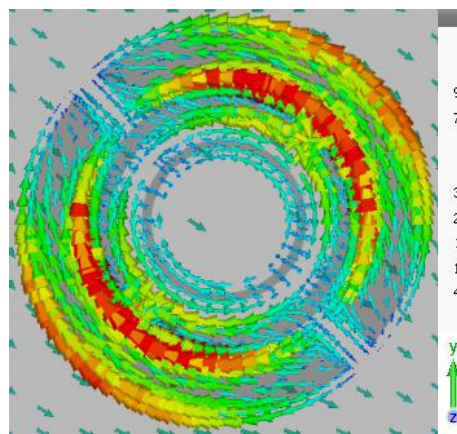

(a)

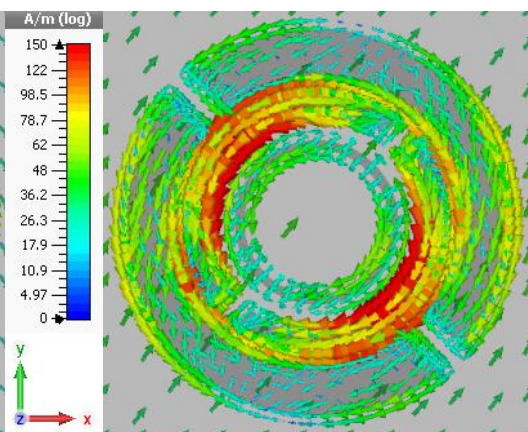

(b)

Fig. 3. Distributions of Surface Current in MMA at frequency (a) $7.9 \mathrm{GHz}$, (b) $11.7 \mathrm{GHz}$.

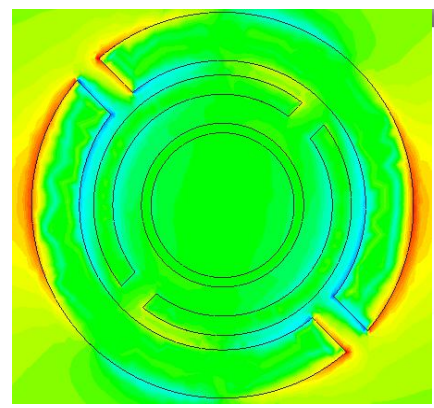

(a)

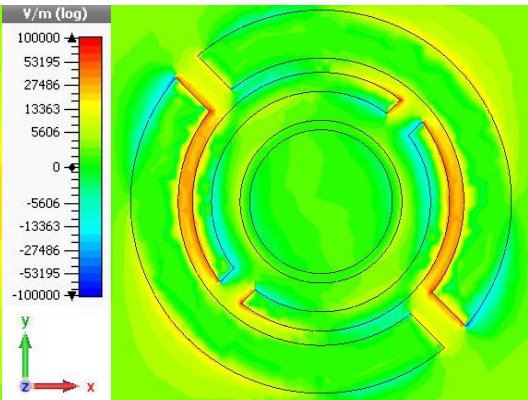

(b)

Fig. 4. Electric field distributions in MMA at frequency (a) $7.9 \mathrm{GHz}$, (b) $11.7 \mathrm{GHz}$.

Brazilian Microwave and Optoelectronics Society-SBMO Brazilian Society of Electromagnetism-SBMag received 10 Feb 2021; for review 26 Feb 2021; accepted 22 May 2021 (c) 2021 SBMO/SBMag (cc) BY 


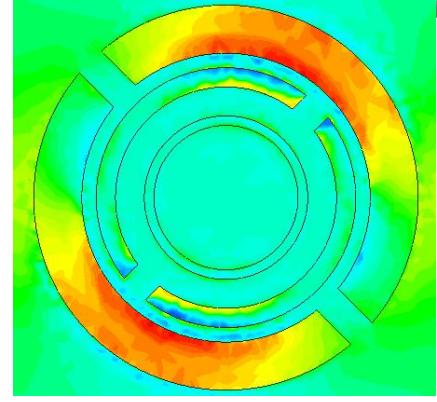

(a)
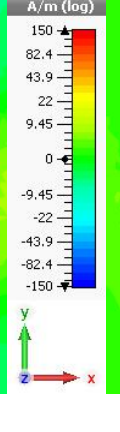

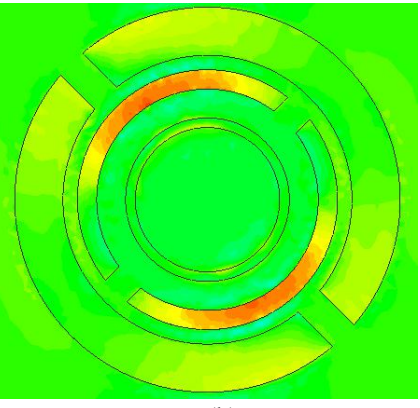

(b)

Fig. 5. Magnetic field distributions in MMA at frequency (a) $7.9 \mathrm{GHz}$, (b) $11.7 \mathrm{GHz}$.

Fig. 3 shows the surface current distributions induced along the three concentric rings of the unit cell of MMA. The direction of induced currents is indicated by the arrow heads and the strength of induced currents by the colour of the arrows. Each ring resonates at a particular resonance band thereby contributing towards the absorption mechanism. The 2D periodic array of unit cells of MMA results in strong coupling of the resonating structure which results in high absorption mechanism of the incident EMR. The surface currents further induce the localized $E$-field and $H$-field responses which get strongly coupled with the incident EMR which results in enhancement of EM field at the resonance frequencies thus the resonant peaks are also contributed by localized electric and magnetic resonances and contributes to complete absorption of incident EMR under impedance matched conditions [17]-[21]. Thus the incident EM energy gets confined in the MMA structure with minimum reflection and maximum absorption.

The $E$-field is aligned along $\mathrm{x}$-axis and $H$-field is aligned along y-axis. From Fig. 3, 4, and 5 it is evident that the individual rings resonator are responsible for high absorption at the distinct resonance peaks and the combined effect of the ring resonators contributes towards the extended wide-band absorption of incident EMR. The surface current is distributed over all the three rings but for first absorption band at $7.9 \mathrm{GHz}$ the distribution of surface current over outermost ring is much denser and for second absorption band at $11.7 \mathrm{GHz}$ the distribution of surface current over middle ring is denser. The surface current distribution for innermost ring is uniform for both resonance peaks.

Similarly, the field distributions are monitored at both the absorption bands. At the first absorption band at $7.9 \mathrm{GHz}$ the electric and magnetic field is highly concentrated at the outermost ring along $x$ axis and $y$-axis respectively whereas at the second absorption band at $11.7 \mathrm{GHz}$ the electric and magnetic field is highly concentrated at the middle ring of the proposed MMA.

To summarize, the absorption at first resonance peak is dominated by the outermost ring whereas absorption at second resonance peak is dominated by the middle ring and the innermost ring has weak contribution towards absorption at both resonance peaks. The concept of non-linear spacing between the rings and the non-linearity in the widths of the rings contributes towards the enhancement of the absorption band extending to $6.32 \mathrm{GHz}$. 


\section{METAMATERIAL PROPERTIES OF MMA UNDER PERFECTLY MATCHED CONDITIONS}

For a perfect metamaterial absorber two conditions must be satisfied. First, the incident EMR must be completely transferred into the MMA which is achieved by perfectly impedance matched conditions and second, the absorbed EMR should be highly dissipated within the MMA which is achieved by high imaginary value of refractive index of MMA for the incident EMR [33]. Fig. 6(a) shows that Re $\left[Z_{\text {eff }}(f)\right]$ is close to 1 and $\operatorname{Im}\left[Z_{\text {eff }}(f)\right]$ is close to 0 , thereby verifying the impedance matched conditions at maximum absorptance peaks.

In order to study the dissipation of EMR inside the material of MMA structure the effective values of $\varepsilon_{\text {eff }}(f), \mu_{\text {eff }}(f)$, and $\eta_{\text {eff }}(f)$ are retrieved from $S_{11}$ and $S_{21}$ parameters using NRW (Nicolson-Ross-Weir) method using (2-4) [34].

$$
\begin{array}{r}
\mu_{e f f}(f)=\frac{2}{j k_{o} d}\left[\frac{1-s_{21}(f)+s_{11}(f)}{1+s_{21}(f)-s_{11}(f)}\right] \\
\varepsilon_{e f f}(f)=\frac{2}{j k_{o} d}\left[\frac{1-s_{21}(f)-s_{11}(f)}{1+s_{21}(f)+s_{11}(f)}\right] \\
\eta(f)=\sqrt{\mu_{e f f}(f) * \varepsilon_{e f f}(f)}
\end{array}
$$

Within the wide-band the $\operatorname{Re}\left[\varepsilon_{\text {eff }}(f)\right]$ and $\operatorname{Re}\left[\mu_{\text {eff }}(f)\right]$ shows Lorentzian transitions and the $\operatorname{Im}\left[\varepsilon_{\text {eff }}(f)\right]$ and $\operatorname{Im}\left[\mu_{\text {eff }}(f)\right]$ attains high value 174.20 and 192.80 at the transition as shown in Fig. 6(b) and Fig. 6(c) respectively. The incident EMR without reflections enters the MMA structure under normalized impedance matched conditions shown in Fig. 6 (a), gets absorbed within the MMA structure as shown in Fig. 2. The intensity of the incident EMR gets attenuated given by (5).

$$
I=I_{o} e^{-2 \alpha z}
$$

where $I_{\mathrm{o}}$ is the intensity of incident EMR and $I$ is the intensity of EMR at distance ' $z$ ', the attenuation constant $\alpha=\eta^{\prime \prime} k$ and the propagation constant $k=2 \pi / \lambda[33] . \eta^{\prime \prime}$ is the imaginary value of refractive index obtained by (4) and $\lambda$ is the wavelength corresponding frequency of maximum absorptance. At frequency $7.9 \mathrm{GHz}$ and 11.7 

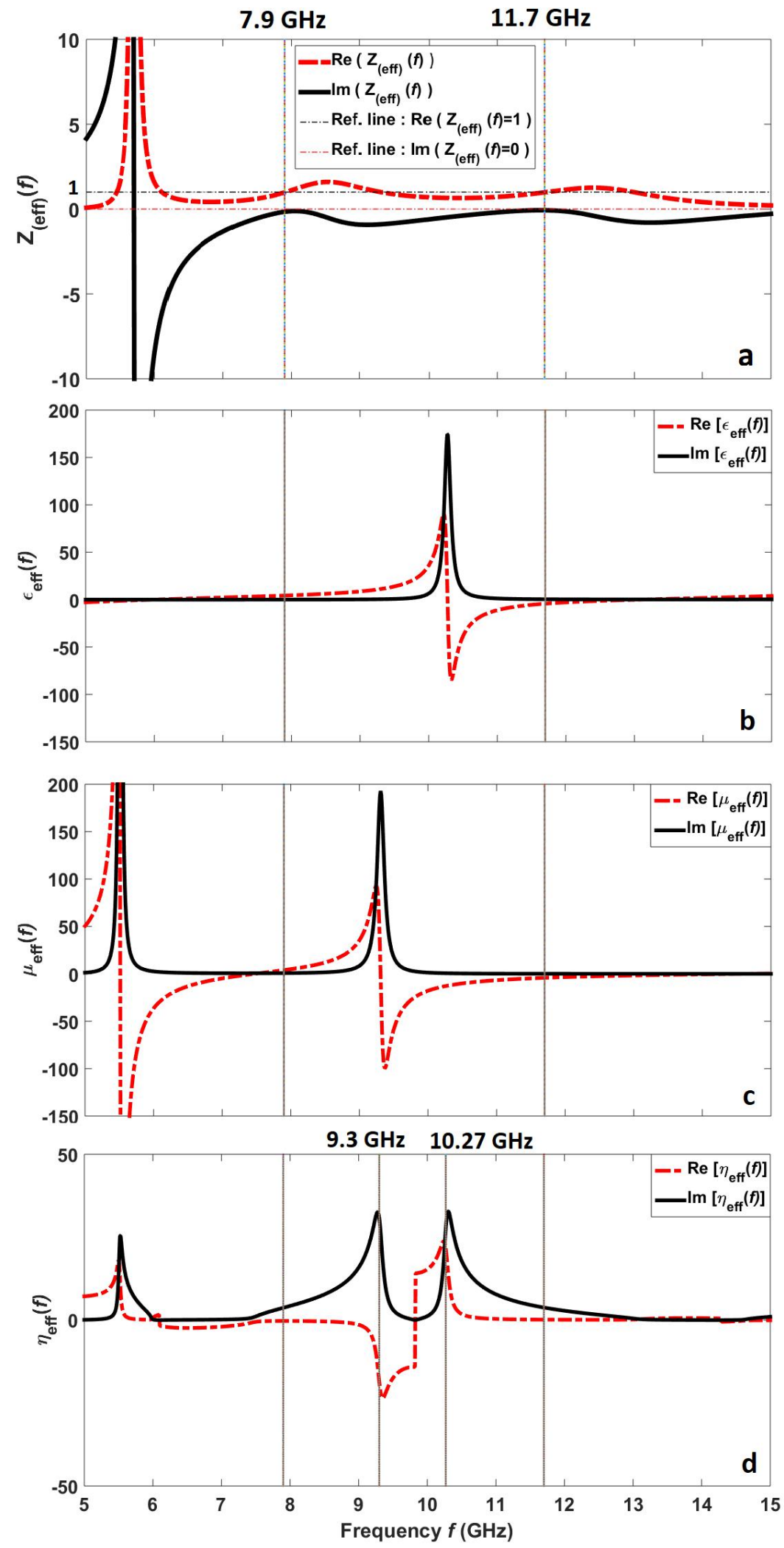

Fig. 6. Simulated values of (a) Effective impedance $Z_{\text {eff }}(f)$. (b) Effective permittivity $\varepsilon_{\text {eff }}(f)$. (c) Effective permeability $\mu_{\text {eff }}(f)$. (d) Effective refractive index $\eta_{\text {eff }}(f)$.

$\mathrm{GHz}$ ' $\alpha$ ' attained the value of $-5.82 \mathrm{~mm}^{-1}$ and $-4.96 \mathrm{~mm}^{-1}$ respectively. The incident EMR which was absorbed maximum at $7.9 \mathrm{GHz}$ and $11.7 \mathrm{GHz}$, the intensity of the EMR after travelling a distance of $\mathrm{z}$ 
Journal of Microwaves, Optoelectronics and Electromagnetic Applications, Vol. 20, No. 2, September 2021 DOI: http://dx.doi.org/10.1590/2179-10742021v20i31207

$\left(=2 \mathrm{t}, \mathrm{t}\right.$ is thickness of the substrate) was reduced to $\mathrm{I} / \mathrm{Io}=5.71 \mathrm{e}^{-21}$ and $4.98 \mathrm{e}^{-18}$ respectively. Thus the absorbed EMR is dissipated inside the material of MMA structure.

\section{EXPERIMENT}

A 2D periodic array of 30 cells $x 30$ cells is fabricated on $441 \mathrm{~cm}^{2}$ FR4 substrate $\left(\varepsilon_{\mathrm{r}}=4.4, \tan \delta=0.02\right)$ of $2 \mathrm{~mm}$ thickness by wet itching process following conventional UV photolithography process [1920] shown in Fig. 7. The MMA is experimentally tested inside an anechoic chamber with the help of identical Horn antennas and Vector network analyzer (Agilent Technologies E5071C, $300 \mathrm{kHz}-20$ GHz) [19], [20].

The distance between the MMA and the horn antennas is carefully fixed so as to diminish the effects of near field on the $S_{11}(f)$ measurements. Under these considerations the distance is approximated to $4.5 \lambda$ which is much larger than $2 \mathrm{D}^{2} / \lambda[35]$ ( $\lambda$ : operating wavelength corresponds to lowest absorption frequency).

To obtain exact results the measurements are taken in two distinct steps. In step 1, the surface of the MMA with 2D array of unit cells (structure side) is positioned to face the horn antennas. The reflection coefficients are measured in $\mathrm{dB}$ scale and saved with the help of VNA. In step 2, the MMA is tilted at $180^{\circ}$ with copper ground plane side to face the horn antennas. Again the reflection coefficients are measured and saved. The difference of the two measured values estimates the corrected measurement in terms of reflection coefficients as shown in Fig. 8. The same procedure is implemented to measure absorptance with variation of angle of incidence $(\theta)$ and polarization angle $(\Phi)$.

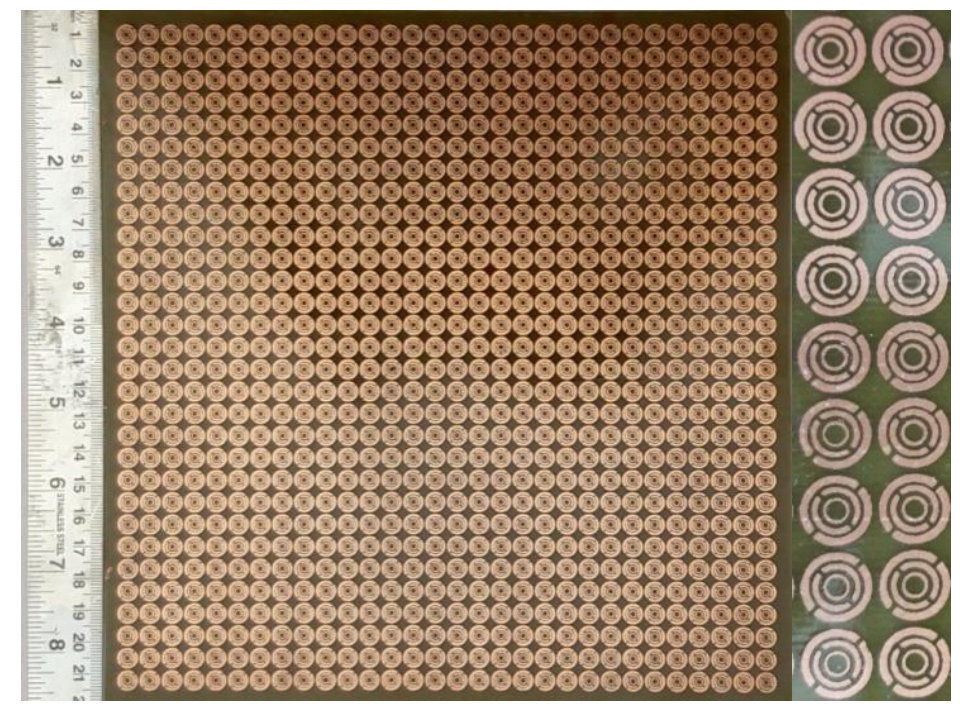

(a)

(b)

Fig. 7. (a) The fabricated MMA with $30 \times 30$ unit cells matrix on $441 \mathrm{~cm}^{2}$ area of FR-4 substrate. (b) Enlarged view of fabricated MMA. 


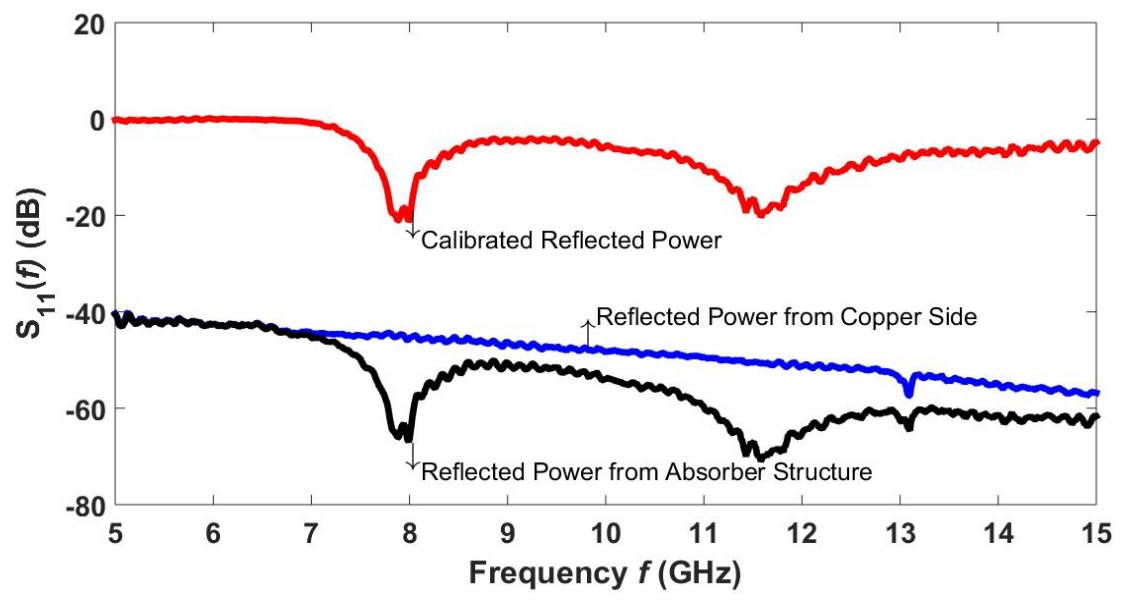

Fig. 8. Measured reflected power as difference of reflected power from structure side and copper side of MMA.

\section{RESULTS AND DISCUSSION}

The $S_{11}(f)$ is measured and recorded with the help of VNA for normal incidence, for variation in $(\theta)$, and $(\Phi)$. Fig. 9(a) and Fig. 9(b) shows the comparison of simulated and experimentally measured values of reflection coefficients, $S_{11}(f)$, and the absorptance, $A(f)$, respectively for normally incident EMR.

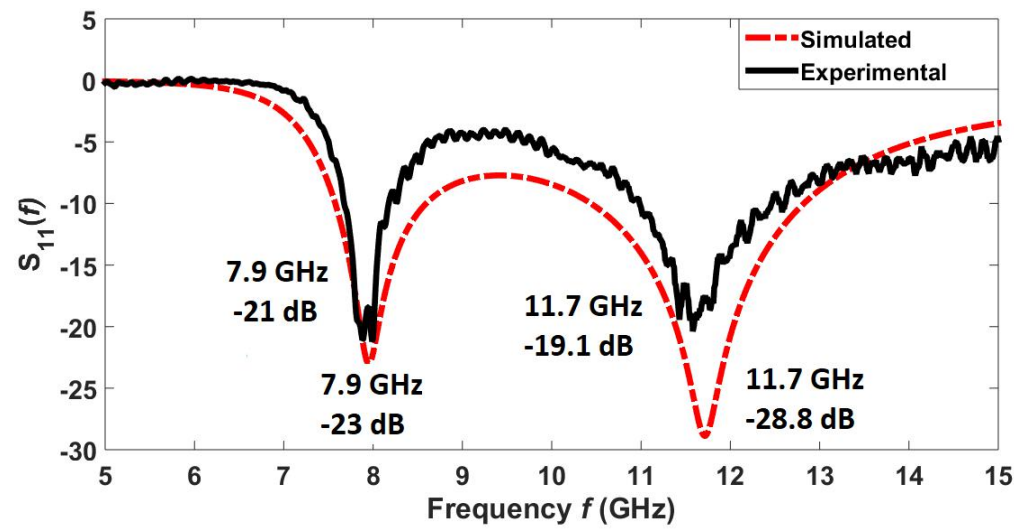

(a)

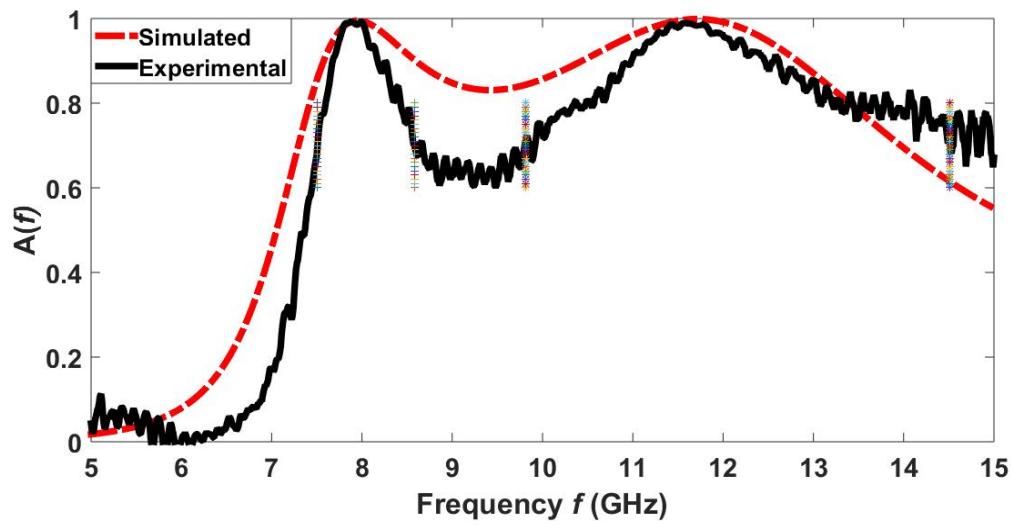

(b)

Fig. 9. Comparison of simulated and experimentally measured values of (a) $S_{11}$ (b) Absorptivities at resonance peaks in proposed MMA. 
Journal of Microwaves, Optoelectronics and Electromagnetic Applications, Vol. 20, No. 2, September 2021 DOI: http://dx.doi.org/10.1590/2179-10742021v20i31207

The simulated and experimentally measured values of $S_{11}(f)$ are in close resemblance at $7.9 \mathrm{GHz}$ and 11.7 $\mathrm{GHz}$ with minor variations in $\mathrm{dB}$ level. The simulated values of reflection coefficients at 7.9 $\mathrm{GHz}$ and $11.9 \mathrm{GHz}$ are $-23 \mathrm{~dB}$ and $-28.8 \mathrm{~dB}$ respectively where as measured values at $7.9 \mathrm{GHz}$ and 11.9 $\mathrm{GHz}$ are $-21 \mathrm{~dB}$ and $-19.1 \mathrm{~dB}$ respectively as shown in Fig. 9(a). The simulated and experimentally measured absorptance peaks have absorptance greater than $99 \%$ at $7.9 \mathrm{GHz}$ and 11.7 GHz. Due to the unique non-linear design structure, the concentric SRRs in the two dimensional array of MMA gets strongly coupled and the absorption peaks get merged to generate FWHM ultra wideband of $6.32 \mathrm{GHz}$ ranging from $7.36 \mathrm{GHz}$ to $13.68 \mathrm{GHz}$ for the normal incidence of EM waves as shown in Fig. 9(b). The simulated and measured average peak to peak absorptance from $7.9 \mathrm{GHz}-$ $11.7 \mathrm{GHz}$ is $91.6 \%$ and $80.1 \%$ respectively. The band of frequencies with desired absorptance greater than $70 \%$ was measured from $7.51 \mathrm{GHz}-8.58 \mathrm{GHz}$ of bandwidth $1.07 \mathrm{GHz}$ [shown in between '+' marks in Fig. 9 (b)] and from $9.81 \mathrm{GHz}-14.51 \mathrm{GHz}$ of bandwidth $4.7 \mathrm{GHz}$ [shown in between '** marks in Fig. 9(b)]. The simulated absorptance dips down to $80 \%$ whereas the experimentally measured value dip is $65 \%$. These minor variations may be contributed due to minor deviation in the ring resonators geometry fabricated by conventional UV lithography and wet etching process.
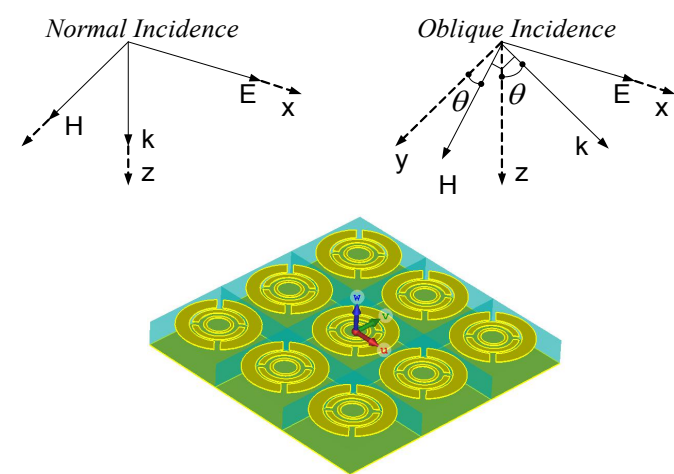

(a)

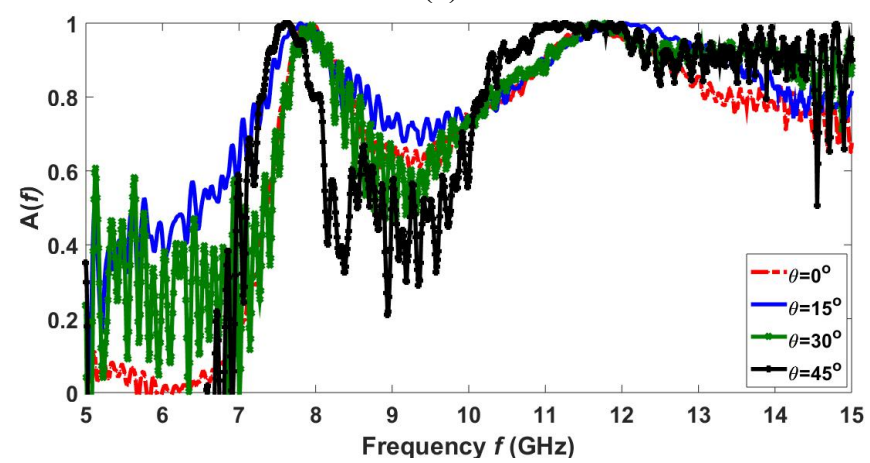

(b)

Fig. 10. (a) Reflection coefficients $S_{11}$ measurement arrangement (b) Experimentally measured Absorptance for normal and varying angle of incidence of EMR.

The MMA is also tested to study its behavior for variation in absorptance with variations in polarization angle $(\Phi)$. Fig. 11(a) and Fig. 11(b) show the arrangement schematic and experimentally measured absorptance at angle of polarization of $0^{\circ}, 45^{\circ}$, and $90^{\circ}$ respectively. 

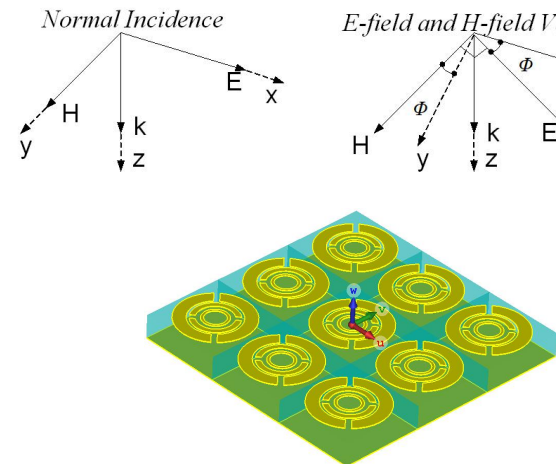

(a)

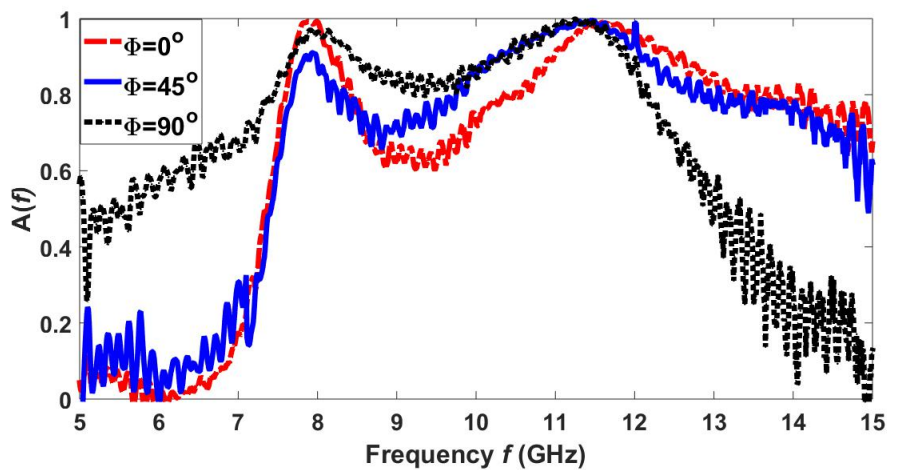

(b)

Fig. 11. (a) Reflection coefficients $S_{11}$ measurement arrangement (b) Experimentally measured Absorptance for varying angle of polarization of incident EMR.

The MMA is tested to study the effect of variation of angle of incidence of EMR on the absorptance. Fig. 10 (a) shows the measurement schematic of absorptance as a function of angle of incidence. The measured absorptance at angle of incidence of $0^{\circ}, 15^{\circ}, 30^{\circ}$, and $45^{\circ}$ is shown in Fig. 10(b). The absorptance for different incident angles $\left(0^{\circ}, 15^{\circ}, 30^{\circ}\right.$, and $\left.45^{\circ}\right)$ is greater than $99 \%$ and the result shows that the absorption capability of proposed MMA is independent to the oblique incidence of EM waves.

The measured absorptance is experimentally found to be greater than $99 \%$. The experimentally measured results show the polarization insensitive behavior of MMA. The insensitive response of proposed MMA to incident and polarization angle $(\theta$ and $\Phi)$ is contributed due to the symmetrical design and strong coupling between ring resonators in the two dimensional array of MMA.

The proposed MMA is compared with reported MMAs consisting ring resonators in Table I. The comparison clearly depicts that the proposed MMA is ultra-thin, polarization insensitive, with appreciable high FWHM and smallest dimension $(7 \mathrm{~mm})$. 
Journal of Microwaves, Optoelectronics and Electromagnetic Applications, Vol. 20, No. 2, September 2021 DOI: http://dx.doi.org/10.1590/2179-10742021v20i31207

TABLE I. COMPARISON OF PROPOSED MMA WITH REPORTED MMAS CONSISTING RING RESONATORS.

\begin{tabular}{|c|c|c|c|c|c|}
\hline MMA & $\begin{array}{c}\text { Centre } \\
\text { Frequency } \\
(\mathbf{G H z})\end{array}$ & $\begin{array}{c}\text { Unit Cell Size } \\
(\mathbf{m m})\end{array}$ & $\begin{array}{l}\text { Thickness } \\
\text { (mm) }\end{array}$ & FWHM & $\begin{array}{r}\text { Polarization } \\
\text { Insensitive }\end{array}$ \\
\hline Lee et al. [36] & 10 & 14.2 & $\begin{array}{c}0.6 \\
\left(0.02 \lambda_{0}\right)\end{array}$ & $11 \%$ & Yes \\
\hline Ghosh et al. [37] & 9.98 & 7.2 & $\begin{array}{c}1 \\
\left(0.033 \lambda_{o}\right)\end{array}$ & $11.52 \%$ & Yes \\
\hline Sood et al. [38] & 5.92 & 9.0 & $\begin{array}{c}1.6 \\
\left(0.031 \lambda_{0}\right)\end{array}$ & $32.77 \%$ & Yes \\
\hline Ghosh et al. [39] & 10.05 & 7.1 & $\begin{array}{c}2 \\
\left(0.067 \lambda_{0}\right)\end{array}$ & $72.64 \%$ & No \\
\hline $\begin{array}{l}\text { Proposed } \\
\text { MMA }\end{array}$ & 9.8 & 7 & $\begin{array}{c}2 \\
\left(0.065 \lambda_{0}\right)\end{array}$ & $64.5 \%$ & Yes \\
\hline
\end{tabular}

\section{CONCLUSION}

A unique ultra-thin and ultra wide-band structure of a MMA is reported. The absorption mechanism and the metamaterial properties of the proposed MMA structure is studied and discussed in terms of simulation and experimentally measured results in detail. The proposed MMA has a uniqueness of non-linear variation in the radius, width and spacing between the rings controlled by parameter $x_{1}$ which makes this design distinct and unique. The absorptance greater than $99 \%$ is simulated and experimentally verified at $7.9 \mathrm{GHz}$ and $11.7 \mathrm{GHz}$. The dual $X$-bands merged to generate FWHM of $6.32 \mathrm{GHz}(64.5 \%)$ extending from $7.36 \mathrm{GHz}$ to $13.68 \mathrm{GHz}$. The average peak to peak simulated and measured absorptance was $91.6 \%$ and $80.1 \%$ respectively. The proposed MMA is tested to study the effect of oblique incidence of EMR (from $0^{\circ}$ to $45^{\circ}$ in steps of $15^{\circ}$ ) and change in angle of polarization $\left(\Phi=0^{\circ}, 45^{\circ}\right.$, and $\left.90^{\circ}\right)$ on the absorptance. The experimentally validated results showed that the proposed MMA structure is insensitive to angle of incidence and polarization that exhibited ultra wide-band due to non-linear variations in the geometry of ring resonators which makes the MMA suitable for reducing RCS and providing stealth technology for defense equipment.

\section{ACKNOWLEDGMENT}

The author(s) acknowledge Dr. K. V. Srivastava, Department of Electrical Eng., IIT Kanpur for providing lab facilities.

\section{REFERENCES}

[1] D. R. Smith , W. J. Padilla, D. C. Vier, S. C. Nemat-Nasser, and S. Schultz, "Composite Medium with Simultaneously Negative Permeability and Permittivity," Physics Review Letters Vol. 84, no. 10, pp. 4184-4187, 1999.

[2] J. B. Pendry, D. Schurig and D. R. Smith, "Controlling electromagnetic fields," Science, vol. 312, pp. 1780-1782, 2006.

[3] V. G. Veselago, "The electrodynamics of substances with simultaneously negative values of $\varepsilon$ and $\mu$," Soviet Physics Uspekhi, vol. 10, no. 4, pp. 509-514, 1968.

[4] J. B. Pendry, A. J. Holden, W. J. Stewart and I. Youngs, "Extremely low frequency plasmons in metallic mesostructures," Physics Review Letters, vol. 76, no. 25, pp. 4773-4776, 1996.

[5] J. B. Pendry, A. J. Holden, D. J. Robbins and W. J. Stewart, "Magnetism from conductors and enhanced nonlinear phenomena," IEEE Transactions on Microwave Theory and Technology, vol. 47, no. 11, pp. 2075-2084, 1999.

Brazilian Microwave and Optoelectronics Society-SBMO received 10 Feb 2021; for review 26 Feb 2021; accepted 22 May 2021 
[6] J. B. Pendry, "Negative refraction makes a perfect lens," Phys. Rev. Lett., vol. 85, no.18, pp. 3966-3969, 2000.

[7] N. Fang, H. Lee, C. Sun and X. Zhang, "Sub-diffraction-limited optical imaging with a silver superlens," Science, vol. 308, pp. 534-537, 2005.

[8] D. Schurig, J. J. Mock, B. J. Justice, S. A. Cummer, J. B. Pendry, A. F. Starr and D. R. Smith, "Metamaterial electromagnetic cloak at microwave frequencies," Science, vol. 314, pp. 977-980, 2006.

[9] R. W. Ziolkowski, "Metamaterial-Based Efficient Electrically Small Antennas," IEEE Transactions on Antennas and Propagation, vol. 54, no. 7, pp. 2113-2130, 2006.

[10] C. R. Williams, M. Misra, S. R. Andrews, S. A. Maier, S. Carretero-Palacios, S. G. Rodrigo, F. J. Garcia-Vidal, and L. Martin-Moreno, "Dual band terahertz wave guiding on a planar metal surface patterned with annular holes," Applied Physics Letters, vol. 96, 011101, 2010.

[11] N. I. Landy, S. Sajuyigbe, J. J. Mock, D. R. Smith, and W. J. Padilla, "Perfect metamaterial absorber," Physical Review Letters, vol. 100, pp. 207402, 2008.

[12] H. Cao, M. Shan, T. Chen, J. Lei, L. Yang, and X. Tan, "Triple-Band Polarization-Independent Ultra-thin Metamaterial Absorber," Progress In Electromagnetics Research M, vol. 77, pp. 93-102, 2019.

[13] K. Tantiwanichapan, A. Ruangphanit, W. Yamwong, R. Meananeatra, A. Srihapat, J. Y. Chia, N. Cota, K. Prasertsuk, P. Rattanawan, C. Thanapirom, R. Jintamethasawat, K. Kasamsook, and N. Klunngien, "Polarization-Independent Wide-Angle Terahertz Metamaterial Absorber: Design, Fabrication and Characterization," Progress In Electromagnetics Research M, vol. 87, pp. 33-42, 2019.

[14] S. Bhattacharyya, S. Ghosh, and K. V. Srivastava, "Triple band polarization-independent metamaterial absorber with bandwidth enhancement at X-band,"Journal of Applied Physics, vol. 114, pp. 094514, 2013.

[15] A. K. Singh, M. P. Abegaonkar, and S. K. Koul, "A Triple Band Polarization Insensitive Ultrathin Metamaterial Absorber for S- C- and X-Bands," Progress In Electromagnetics Research M, Vol. 77, 187-194, 2019.

[16] B. X. Wang, L. L. Wang, G. Z. Wang, W. Q. Huang, X. F. Li, and X. Zhai, "Theoretical Investigation of Broad-band and Wide-Angle Terahertz Metamaterial Absorber,” IEEE Photonics Technology Letters, vol. 26, no. 2, pp. 111- 114, Jan. 15, 2014.

[17] T. Wu, Y. M. Ma, J. Chen, and L. L. Wang, "A Low Profile Quadruple-Band Polarization Insensitive Metamaterial Absorber," Progress In Electromagnetics Research M, Vol. 90, 69-79, 2020.

[18] M. Agarwal, A. K. Behera and M. K. Meshram, "Wide-angle quad-band polarization insensitive metamaterial absorber," Electronics Letters, vol. 52, no.5, pp. 340-342, 2016.

[19] A. Agrawal, M. Misra, and A. Singh "Oblique Incidence and Polarization Insensitive Multiband Metamaterial Absorber with Quad Paired Concentric Continuous Ring Resonators" Progress In Electromagnetics Research M, vol. 60, pp. 33-46, 2017.

[20] A. Agrawal, M. Misra, and A. Singh, "Wide incidence angle and polarization insensitive dual broad-band metamaterial absorber based on concentric split and continuous rings resonator structure, ” Materials Research Express, vol. 5, no. 11, pp. 115801, 2018.

[21] F. Dincer, M. Karaaslan, S. Colak, E. Tetik, O. Akgol, O. Altıntas, and C. Sabah, "Multiband polarization independent cylindrical metamaterial absorber and sensor application," Modern Physics Letters B, Vol. 30, No. 8, 1650095, 2016.

[22] L. Huang and H. Chen, "Multi-band and polarization insensitive metamaterial absorber," Progress In Electromagnetics Research, vol. 113, pp. 103-110, 2011.

[23] M. H. Li, H. L. Yang, and X. W. Hou, "Perfect metamaterial absorber with dual bands," Progress In Electromagnetics Research, vol. 108, pp. 37- 49, 2010.

[24] A. K. Singh, M. P. Abegaonkar, and S. K. Koul, "A Triple Band Polarization Insensitive Ultrathin Metamaterial Absorber for S- C- and X-Bands", Progress In Electromagnetics Research M, vol. 77, pp. 187-194, 2019.

[25] W. Li and J. Valentine, "Metamaterial Perfect Absorber Based Hot Electron Photodetection", Nano Lett., vol. 14 , pp. 3510-3514, 2014

[26] H. Huang, Y. Liu, S. Zhang and S. Gong, "Multiband Metamaterial- Loaded Monopole Antenna for WLAN/WiMAX Applications," IEEE Antennas and Wireless Propagation Letters, vol. 14, pp. 662-665, 2015.

[27] D. Langley, R. A. Coutu, Jr, L. A. Starman, and S. Rogers, "Optical metamaterials for photonics applications", Proceedings of SPIE - The International Society for Optical Engineering, vol. 7468, pp. 74680H1-8, 2009.

[28] P. Kung and S. M. Kim, "Terahertz metamaterial absorbers for sensing and imaging", Progress in Electromagnetics Research Symposium, pp. 232-235, 2013.

[29] D. Wen, H. Yang, Q. Ye, M. Li, L. Guo, and J. Zhang, "Broadband metamaterial absorber based on a multi-layer structure," Physica Scripta, vol. 88, pp. 015402 (17), 2013.

[30] W. Yuan and Y. Cheng, "Low-frequency and broadband metamaterial absorber based on lumped elements: design, characterization and experiment," Applied Physics A, vol. 117, pp. 1915-1921, 2014.

[31] H. Kumar and G. Kumar, "A Broadband Planar Modified Quasi-Yagi Using Log-Periodic Antenna”, Progress In Electromagnetics Research Letters, vol. 73, 23-30, 2018.

[32] D. R. Dykaar, B. I. Greene, J. F. Federici, A. F. J. Levi, L. N. Pfeiffer, and R. F. Kopf, "Log-periodic antennas for pulsed terahertz radiation", Appied Physics Letters, vol. 59, 1991.

[33] K. Bhattarai, S. Silva, K. Song, A. Urbas, S. J. Lee, Z. Ku, and J. Zhou, "Metamaterial Perfect Absorber Analyzed by a Meta-cavity Model Consisting of Multilayer Metasurfaces,” Scientific Reports Nature, vol. 7, no. 10569, 2017. 
Journal of Microwaves, Optoelectronics and Electromagnetic Applications, Vol. 20, No. 2, September 2021 DOI: http://dx.doi.org/10.1590/2179-10742021v20i31207

[34] O. Luukkonen, S. I. Maslovski, and S. A. Tretyakov, "A stepwise Nicolson-Ross-Weir based material parameter extraction method," IEEE Antenna and Wireless Propagation Letters, vol. 10, 1295-1298, 2011.

[35] C. A Balanis, Antenna Theory: Analysis and Design, 3rd Edition, Ch. 2, 34, 2005.

[36] J. Lee and S. Lim, "Bandwidth-enhanced polarization insensitive microwave metamaterial using double resonance", Electronics Letters, Vol. 47, pp. 8-9, 2011.

[37] S. Ghosh, S. Bhattacharyya, Y. Kaiprath, and K. V. Srivastava, "Bandwidth enhanced polarization insensitive microwave metamaterial absorber and it's equivalent circuit model”, Journal of Applied Phyics, Vol. 115, pp. 104503, 2014.

[38] D. Sood and C. C. Tripathi, “A Wideband Wide-Angle Ultra-Thin Metamaterial Microwave Absorber”, Progress In Electromagnetics Research M, Vol. 44, pp. 39-46, 2015.

[39] S. Ghosh, S. Bhattacharyya, D. Chaurasiya, and K. V. Srivastava, "An ultra wideband ultra thin metamaterial absorber based on circular split rings," IEEE Transactions on Antennas and Propagation, Vol. 14, pp. 1172-1175, 2015. 